\title{
Editorial: Foundations of Utility and Risk Conference (FUR 2018)
}

\author{
John D. Hey ${ }^{1} \cdot$ Chris Starmer $^{2}$ (D) \\ Published online: 25 November 2019 \\ (c) Springer Science+Business Media, LLC, part of Springer Nature 2019
}

One of the great delights in organising a conference in the Foundations of Utility and Risk conference series is the heterogeneity of the presentations. Since the first meeting in 1982, the conference has broadened its domain and, while the common theme of understanding human decision-making runs through the work submitted, there was a great diversity in the submitted papers which included theoretical pieces, experiments and other empirical studies. There was also considerable heterogeneity in the expertise, training and interests of participants and presenters. Hence, as FUR attendees will have come to expect, the 2018 conference in York was an intellectually diverse and fascinating event.

We were delighted to have been invited to edit this Special Issue of Theory and Decision for papers presented at the conference and we are grateful to all the authors who submitted their work for consideration. The selection was not an easy task as space constraints inevitably mean that not all worthy papers make the final cut. It has taken us some time, but finally we have a set of papers that we are proud to present in this Special Issue.

The papers included reflect the heterodox nature of the FUR event: we have a theoretical paper, several experimental studies, plus a paper analysing published empirical data. We have papers on individuals and on games, though none, unfortunately, on markets. The papers also show a mix of focus on foundational topics through to more applied topics, as well as diversity in their statistical methodology; the majority employing descriptive statistics, and carrying out comparative static tests across the subjects; one estimating preference functionals, and estimating subject by subject.

This foreword is intended to provide a brief introduction to what is to follow, and to do this we have to discuss them in some order. Our ranking of the papers should not be inferred from this ordering - indeed, we aimed at neutrality by sequencing

Chris Starmer

chris.starmer@nottingham.ac.uk

1 Department of Economics and Related Studies, University of York, Heslington, York YO10 5DD, UK

2 School of Economics, University of Nottingham, Nottingham NG7 2RD, UK 
them loosely by genre. We start with the one theory paper, then move on to the studies on individual choice and conclude with the game-theoretic pieces. We discuss each of them only very briefly, offering no more than quick tasters to whet the appetite. Indeed, we encourage readers to get quickly to the main dishes themselves.

The paper by Embrey, "States of Nature and States of Mind: A Generalised Theory of Decision-Making", is a contribution to theory 'after' (or 'beyond') Expected Utility (EUT) theory. He proposes a generalisation of EUT in which decision makers may either follow EUT or "act impulsively". In other words, they have "heterogeneous thought processes". This latter is defined and its implications explored in a series of examples, with a particular focus on the dynamic consequences of the model. FUR has traditionally been a place to air new, behaviourally oriented, theories of decision under risk. This paper continues that tradition in a thought-provoking way. We do not think that this is the end of the story by any means, but it demonstrates the still rich potential for development of new models in this area.

The three papers on individual decision making are quite heterogeneous, in the questions they address, in their methodologies and in the types of data that they use. The paper by Bacon, Conte and Moffatt, "A test of Risk Vulnerability in the Wider Population", uses data from the German SOEP (Socio-Economic Panel Survey) to test for 'Risk Vulnerability' in a more heterogeneous, and representative, sample of the population than is normally the case in experiments. Risk Vulnerability-a concept closely related to decreasing absolute risk aversion-is the phenomenon of an individual becoming less willing to take risk when there is an increase in "background risk", with background risk in this study being proxied by a standard measure of stock market volatility in Germany. In an analysis across respondents, they find evidence for the existence of risk vulnerability in the SOEP. The paper also makes a contribution in identifying a new way to quantify risk vulnerability.

The next two papers we mention both build on contemporary work which has identified apparent "imprecision" in individual preferences. The paper by Jakubczyk, "Elicitation and Modelling of Imprecise Utility of Health States", uses data from a questionnaire. A typical question is the following: "Imagine you may live for 10 years in a wheelchair or $T$ years in full health (both followed by sudden and painless death). What $T$ would make you exactly indifferent?" An interesting feature of this study was that respondents were allowed to be imprecise about their value for $T$ (they could report a range). The author interprets this as the subjects being imprecise in their preferences and proposes to model the imprecision through an approach exploiting fuzzy sets. The paper explores both how features of health states might contribute to explaining the extent of the imprecision and whether revealed imprecision may help account for anomalies in application of the time trade-off method for health state evaluation.

The paper by Permana, "Why Do People Prefer Randomisation: An Experimental Investigation", uses experimental data to explore why subjects, when asked to choose between two options, reply 'I am not sure', when the implications are that the experimenter will randomise between the two options. While Permana uses a setup similar to earlier work examining imprecision, the incentive mechanism applied in this experiment means that, saying 'I am not sure' is most naturally interpreted as expressing a desire for randomisation, and not as indicating an imprecise preference. 
Permana proposes four different models to account for this preference for randomisation and fits them subject by subject finding that two emerge with more support: one is a model embodying a preference for randomisation, the other embodies a threshold below which the chooser cannot discriminate between options.

The remaining three papers are all conducted with two-player games but differ in the questions being asked and the games being played. The paper by Angelovski, Di Cagno and Guth, "Telling the Other What One Knows? Strategic Lying in a Modified Acquiring-a-Company Experiment with Two-Sided Private Information", explores whether subjects lie about the information that they privately possess, and can pass to the other. Their setup has two key modifications relative to the original Acquiring-a-Company game: one is the two-sided nature of the private information in which both buyers and sellers hold value-relevant information; the other is the use of cheap talk communication. It turns out that these features provide purchase on several interesting hypotheses. They also explore the effect of gender on behaviour.

The paper by Attanasi, Concina, Kamate and Rotondi, "Firm's protection against disasters: are investment and insurance substitutes or complements?", uses a twoplayer experimental game to examine whether firms take out insurance against some adverse event, or invest in technology to reduce the probability of it. While there is some time dependence, they conclude that the two are generally substitutes.

The paper by Chowdhury and Karakostas, "An Experimental Investigation of the 'Tenuous Trade-off' between Risk and Incentives in Organizations", uses a Principal/Agent experimental game to explore the relationship between risk and incentives. A primary finding is that principals offer higher fixed wages and lower piece rates in the presence of risk. In addition, the relationships between effort and reward are consistent with positive reciprocity.

The papers in this Special Issue not only reveal the ongoing strength of the FUR organisation, but also its ability in provoking economists, psychologists and others to bring decision sciences to bear on many of the burning questions of the day through diverse and imaginative approaches. We thank all the authors for their contributions, and the Journal for giving us this opportunity to oversee this volume.

Publisher's Note Springer Nature remains neutral with regard to jurisdictional claims in published maps and institutional affiliations. 